\title{
Axial and focal-plane diffraction catastrophe integrals
}

\author{
M V Berry ${ }^{1}$ and C J Howls ${ }^{2}$ \\ ${ }^{1}$ H H Wills Physics Laboratory, Tyndall Avenue, Bristol BS8 1TL, UK \\ 2 School of Mathematics, University of Southampton, Southampton, SO17 1BJ, UK
}

Received 22 April 2010

Published 2 August 2010

Online at stacks.iop.org/JPhysA/43/375206

\begin{abstract}
Exact expressions in terms of Bessel functions are found for some of the diffraction catastrophe integrals that decorate caustics in optics and mechanics. These are the axial and focal-plane sections of the elliptic and hyperbolic umbilic diffraction catastrophes, and symmetric elliptic and hyperbolic unfoldings of the $X_{9}$ diffraction catastrophes. These representations reveal unexpected relations between the integrals.
\end{abstract}

PACS numbers: $\quad$ 02.30.Gp, 02.30.Uu, 02.40.Xx, 02.60.Jh, 42.25.Fx

\section{Introduction}

In optics and mechanics, caustics are described mathematically by the singularities of catastrophe theory [1], and decorated by interference patterns that have been extensively studied not only numerically but also experimentally [2-7] (for example in the optics of liquid-droplet lenses). These are the diffraction catastrophes [8], described mathematically by oscillatory integrals. They form a hierarchy, of which the first member is the Airy function $[9,10]$, expressible in terms of the more familiar Bessel functions [11]:

$$
\left.\begin{array}{rlr}
\operatorname{Ai}(z) & =\frac{1}{2 \pi} \int_{-\infty}^{\infty} \mathrm{d} s \exp \left\{\mathrm{i}\left(\frac{1}{3} s^{3}+z s\right)\right\} & \\
& =\frac{\sqrt{z}}{\pi \sqrt{3}} K_{1 / 3}\left(\frac{2}{3} z^{3 / 2}\right) & (z \geqslant 0) \\
& =\frac{\sqrt{|z|}}{3}\left(J_{-1 / 3}\left(\frac{2}{3}|z|^{3 / 2}\right)+J_{1 / 3}\left(\frac{2}{3}|z|^{3 / 2}\right)\right) & (z<0)
\end{array}\right\} .
$$

Beyond the Airy function, higher diffraction catastrophes form a new class of special functions [12]. Current knowledge of them is summarised in chapter 36 of the recently-released Digital Library of Mathematical Functions [13]. The functions have applications throughout wave physics, and form the skeletons of uniform asymptotic approximations.

The full unfoldings of diffraction catastrophes cannot be expressed in more elementary terms. However, special sections of some higher catastrophes, important because they 
correspond to geometric symmetries, can be expressed in terms of Bessel functions, and our purpose here is to demonstrate this. Such reduction to known functions is useful, not only to get analytical insight into the structure of the diffraction patterns but also to supplement numerical computations, which for these oscillatory integrals become unstable or time-consuming far from the most singular points. The formulas we present here can be regarded as supplementing those assembled in chapter 36 of [13].

The possibility of simplification was suggested by a known relation for the Pearcey function [14]

$$
\Psi_{2}(x, z)=\int_{-\infty}^{\infty} \mathrm{d} s \exp \left\{\mathrm{i}\left(s^{4}+z s^{2}+x s\right)\right\}
$$

that decorates the cusp caustic: on the symmetry axis $x=0$,

$$
\begin{aligned}
\Psi_{2}(0, z)=\frac{\pi}{2} & \sqrt{\frac{|z|}{2}} \exp \left(-\frac{1}{8} \mathrm{i} z^{2}\right) \\
& \times\left(\exp \left(\frac{1}{8} \mathrm{i} \pi\right) J_{-1 / 4}\left(\frac{1}{8} z^{2}\right)-\operatorname{sgn} z \exp \left(-\frac{1}{8} \mathrm{i} \pi\right) J_{1 / 4}\left(\frac{1}{8} z^{2}\right)\right) .
\end{aligned}
$$

The singularities whose special sections we discuss here depend on three parameters $x, y, z$. These are the elliptic umbilic $[6,8]$

$$
\Psi_{\mathrm{E}}(x, y, z)=\int_{-\infty}^{\infty} \mathrm{d} s \int_{-\infty}^{\infty} \mathrm{d} t \exp \left\{\mathrm{i}\left(s^{3}-3 s t^{2}+z\left(s^{2}+t^{2}\right)+y t+x s\right)\right\},
$$

the hyperbolic umbilic [5]

$$
\begin{aligned}
& \Psi_{\mathrm{H}}(x, y, z)=\int_{-\infty}^{\infty} \mathrm{d} s \int_{-\infty}^{\infty} \mathrm{d} t \exp \left\{\mathrm{i}\left(s^{3}+t^{3}+z s t+y t+x s\right)\right\} \\
& =2^{1 / 3} \int_{-\infty}^{\infty} \mathrm{d} \mu \int_{-\infty}^{\infty} \mathrm{d} v \exp \left\{\mathrm{i}\left(u^{3}+3 u v^{2}+\frac{z\left(u^{2}-v^{2}\right)}{2^{2 / 3}}+\frac{(x+y) u+(x-y) v}{2^{1 / 3}}\right)\right\},
\end{aligned}
$$

and their counterparts in the symmetrical partial unfoldings of the codimension- 9 singularity $\mathrm{X}_{9}$ :

$X_{\mathrm{E}}(x, y, z)=\int_{-\infty}^{\infty} \mathrm{d} s \int_{-\infty}^{\infty} \mathrm{d} t \exp \left\{\mathrm{i}\left(s^{4}+t^{4}-6 s^{2} t^{2}+z\left(s^{2}+t^{2}\right)+y t+x s\right)\right\}$

[7] and

$$
\begin{aligned}
X_{\mathrm{H}}(x, y, z) & =\int_{-\infty}^{\infty} \mathrm{d} s \int_{-\infty}^{\infty} \mathrm{d} t \exp \left\{\mathrm{i}\left(s^{4}+t^{4}+6 s^{2} t^{2}+z\left(s^{2}-t^{2}\right)+y t+x s\right)\right\} \\
& =2^{-1 / 2} \int_{-\infty}^{\infty} \mathrm{d} \mu \int_{-\infty}^{\infty} \mathrm{d} v \exp \left\{\mathrm{i}\left(u^{4}+v^{4}+2^{1 / 2} u v z+\frac{(x+y) u+(x-y) v}{2^{3 / 4}}\right)\right\}
\end{aligned}
$$

Figure 1 shows the caustic surfaces of the four singularities. These and other diffraction catastrophes have been extensively explored experimentally [3].

We will study $\Psi_{\mathrm{E}}, \Psi_{\mathrm{H}}, X_{\mathrm{E}}$ and $X_{\mathrm{H}}$ along the symmetry axis $(0,0, z)$ (section 2$)$ and in the focal plane $(x, y, 0)$ (section 3 ). For clarity, we present the results in the main text and outline the derivations of the axial formulas of section 2 in appendix A, and the focal-plane formulas of section 3 in appendix B.

There are alternative symmetric hyperbolic unfoldings of $X_{9}$, obtained by replacing $u v$ by $u^{2}+v^{2}$ or $u^{2}-v^{2}$ in (1.7), listed in [3]; we do not study these further, because for all $x, y, z$ the integrals factorise into products of Pearcey functions (1.2). 

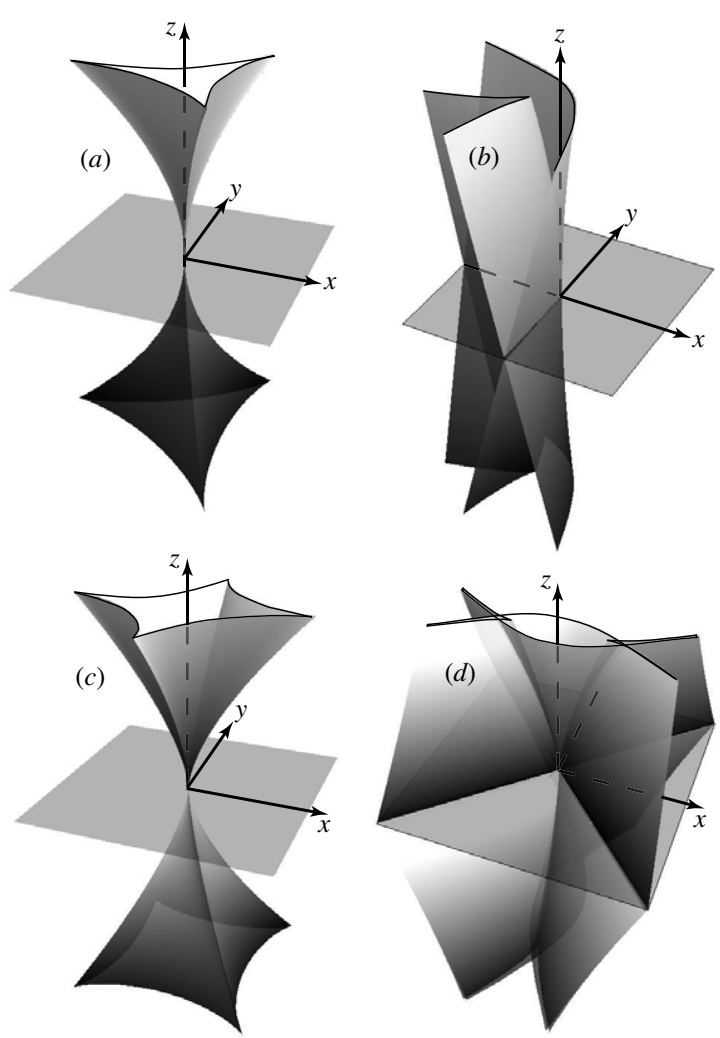

Figure 1. Caustic surfaces, indicating the axial direction $z$ and the focal plane $\{x, y\}$. (a) Elliptic umbilic; (b) hyperbolic umbilic; $(c)$ elliptic section of $X_{9} ;(d)$ hyperbolic section of $X_{9}$.

There is some overlap between the description of caustics in terms of catastrophe theory, which lists the singularities that are stable under perturbation, and the classical theory of wavefront aberrations The connection was established long ago [2], and we do not revisit it here.

\section{Axial integrals}

For the elliptic umbilic (1.4), the $z$ axis lies within the trumpet-shaped caustic surface [6] (bifurcation set) (figure 1(a)), whose three sheets coincide at the focal point $z=0$. On the axis,

$$
\begin{aligned}
\Psi_{\mathrm{E}}(0,0, z) & =\int_{-\infty}^{\infty} \mathrm{d} s \int_{-\infty}^{\infty} \mathrm{d} t \exp \left\{\mathrm{i}\left(s^{3}-3 s t^{2}+z\left(s^{2}+t^{2}\right)\right)\right\} \\
& =2 \pi \int_{0}^{\infty} \mathrm{d} r r J_{0}\left(r^{3}\right) \exp \left\{\mathrm{i} z r^{2}\right\} \\
& =2 \pi \sqrt{\frac{\pi z}{27}} \exp \left\{\frac{2}{27} \mathrm{i} z^{3}\right\}\left(J_{-1 / 6}\left(\frac{2}{27} z^{3}\right)+\mathrm{i} J_{1 / 6}\left(\frac{2}{27} z^{3}\right)\right)(z \geqslant 0) .
\end{aligned}
$$

The second equality results from expressing the integral over the $s, t$ plane in polar coordinates. The third equality is the new result; the extension to $z<0$ follows from the symmetry 

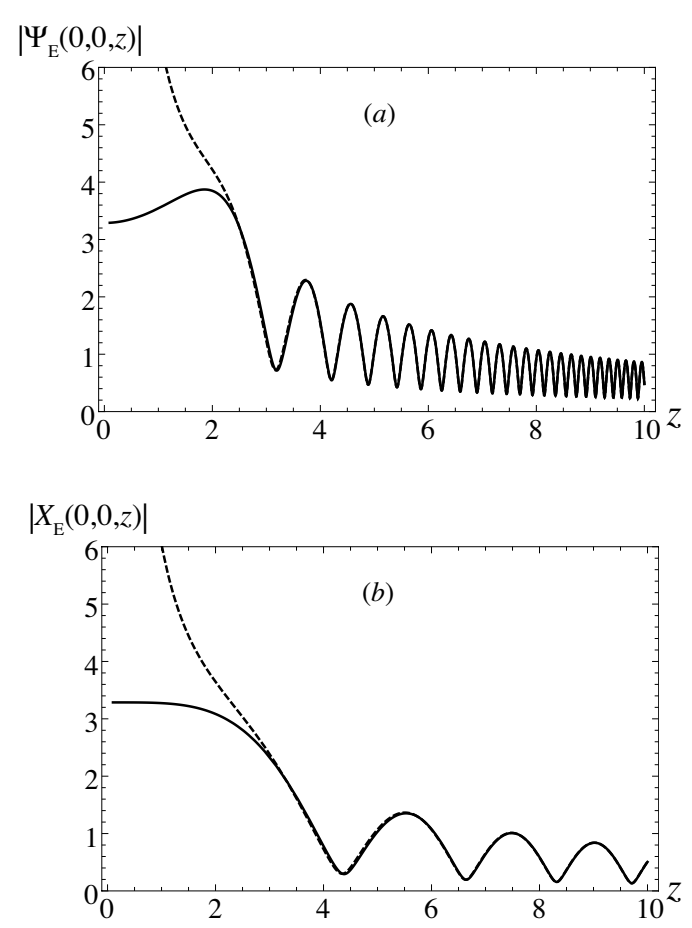

Figure 2. (a) Axial Elliptic umbilic diffraction catastrophe $\left|\Psi_{\mathrm{E}}(0,0, z)\right|$; full curve, exact formula (2.1); dashed curve, approximation (2.2). (b) Axial Elliptic $X_{9}$ diffraction catastrophe $\left|X_{\mathrm{E}}(0,0, z)\right|$; full curve, exact formula (2.4); dashed curve, approximation (2.5). The corresponding pictures for $\left|\Psi_{\mathrm{H}}(0,0, z)\right|$ and $\left|X_{\mathrm{H}}(0,0, z)\right|$ would look identical, apart from the scalings embodied in the last lines of (2.3) and (2.6).

$\Psi_{\mathrm{E}}(x, y,-z)=\Psi_{\mathrm{E}}^{*}(x, y,-z)$ (equation (3.2.24 of [13]). Away from the focus, elementary Bessel asymptotics gives

$$
\Psi_{\mathrm{E}}(0,0, z) \approx \frac{\mathrm{i} \pi}{z}\left[1+\sqrt{3} \exp \left(\frac{4}{27} \mathrm{i} z^{3}-\frac{1}{2} \mathrm{i} \pi \operatorname{sgn} z\right)\right],
$$

as illustrated in figure $2(a)$.

The bifurcation set of the hyperbolic umbilic is very different: the $z$ axis lies between the smooth and cusped caustic sheets (figure $1(b)$ ). Therefore it is surprising that the axial diffraction is essentially the same as (2.2):

$$
\begin{aligned}
\Psi_{\mathrm{H}}(0,0, z) & =\int_{-\infty}^{\infty} \mathrm{d} s \int_{-\infty}^{\infty} \mathrm{d} t \exp \left\{\mathrm{i}\left(s^{3}+t^{3}+z s t\right)\right\} \\
& =\frac{2 \pi}{3^{1 / 3}} \int_{-\infty}^{\infty} \mathrm{d} t \exp \left\{\mathrm{i} t^{3}\right\} \operatorname{Ai}\left(\frac{z t}{3^{1 / 3}}\right) \\
& =\frac{2}{9} \pi \sqrt{\pi z} \exp \left\{\frac{1}{54} \mathrm{i} z^{3}\right\}\left(J_{-1 / 6}\left(\frac{1}{54} z^{3}\right)-\mathrm{i} J_{1 / 6}\left(\frac{1}{54} z^{3}\right)\right)(z \geqslant 0) \\
& =\frac{2^{1 / 3}}{\sqrt{3}} \exp \left(\frac{1}{27} \mathrm{i} z^{3}\right)\left[\Psi_{\mathrm{E}}\left(0,0, \frac{z}{2^{2 / 3}}\right)\right]^{*} .
\end{aligned}
$$

Here the second equality follows from the first after evaluating the $s$ integral. The third equality is the new result; the extension to $z<0$ follows from the symmetry 
$\Psi_{\mathrm{H}}(x, y,-z)=\Psi_{\mathrm{H}}^{*}(x, y,-z)$ (equation (3.2.24 of [13]). The fourth equality, also new, is the explicit relation to the axial elliptic umbilic. This unexpected connection was suggested by numerical calculations, after which we demonstrated it analytically.

For the elliptic partial unfolding (1.6) of $X_{9}$, the $z$ axis also lies within a trumpet-shaped caustic surface [7] (bifurcation set) (figure 1(c)), with four sheets (rather than three as in the elliptic umbilic) coinciding at the focal point $z=0$. On the axis,

$$
\begin{aligned}
X_{\mathrm{E}}(0,0, z) & =\int_{-\infty}^{\infty} \mathrm{d} s \int_{-\infty}^{\infty} \mathrm{d} t \exp \left\{\mathrm{i}\left(s^{4}+t^{4}-6 s^{2} t^{2}+z\left(s^{2}+t^{2}\right)\right)\right\} \\
& =\pi \int_{-\infty}^{\infty} \mathrm{d} \rho \exp (\mathrm{i} z \rho) J_{0}\left(\rho^{2}\right) \\
& =\frac{\pi^{2}}{8}|z|\left[J_{-1 / 4}\left(\frac{1}{8} z^{2}\right)^{2}-J_{1 / 4}\left(\frac{1}{8} z^{2}\right)^{2}+\mathrm{i} \operatorname{sgn}(z) \sqrt{2} J_{-1 / 4}\left(\frac{1}{8} z^{2}\right) J_{1 / 4}\left(\frac{1}{8} z^{2}\right)\right] .
\end{aligned}
$$

The second equality results from expressing the integral over the $s, t$ plane in polar coordinates and replacing the radial coordinate by its square root, and the third equality is the new result. Away from the focus, elementary Bessel asymptotics gives

$$
X_{\mathrm{E}}(0,0, z) \approx \frac{\mathrm{i} \pi}{z}\left[1+\sqrt{2} \exp \left\{\mathrm{i} \operatorname{sgn} z\left(\frac{1}{4} z^{2}-\frac{1}{2} \pi\right)\right\}\right],
$$

as illustrated in figure $2(b)$.

For the hyperbolic partial unfolding (1.7) of $X_{9}$, whose bifurcation set is illustrated in figure $1(d)$, the axial diffraction catastrophe is

$$
\begin{aligned}
X_{\mathrm{H}}(0,0, z) & =\int_{-\infty}^{\infty} \mathrm{d} s \int_{-\infty}^{\infty} \mathrm{d} t \exp \left\{\mathrm{i}\left(s^{4}+t^{4}+6 s^{2} t^{2}+z\left(s^{2}-t^{2}\right)\right)\right\} \\
& =8 \int_{0}^{\infty} \mathrm{d} u u \cos \left(z u^{2}\right) \int_{0}^{\infty} \mathrm{d} v \exp \left\{\mathrm{i} u^{4} \cosh (4 v)\right\} \\
& =\exp \left(\frac{1}{4} \mathrm{i} \pi\right) \int_{0}^{\infty} \mathrm{d} \rho \cos \left(z \rho \exp \left(\frac{1}{4} \mathrm{i} \pi\right)\right) K_{0}\left(\rho^{2}\right) \\
& =\frac{\pi^{2}}{8 \sqrt{2}} \exp \left(\frac{1}{4} \mathrm{i} \pi\right)|z|\left(J_{-1 / 4}\left(\frac{1}{8} z^{2}\right)^{2}-\mathrm{i} J_{1 / 4}\left(\frac{1}{8} z^{2}\right)^{2}\right) \\
& =-\frac{\exp \left(\frac{1}{4} \mathrm{i} \pi\right)}{\sqrt{2}}\left[\frac{H_{1 / 4}^{(2)}\left(\frac{1}{8} z^{2}\right)}{H_{1 / 4}^{(1)}\left(\frac{1}{8} z^{2}\right)}\right] X_{\mathrm{E}}(0,0, z) .
\end{aligned}
$$

The second equality follows from expressing the integration over an octant in the $s, t$ plane in hyperbolic coordinates $u, v$, and the third equality from evaluating the $v$ integral and the replacement $u^{4}=\mathrm{i} \rho^{2}$. The fourth equality is the new result, and the fifth is the explicit connection with the axial elliptic $X_{9}$ formula (2.4).

\section{Focal-plane integrals}

The explicit expression for the elliptic umbilic (1.4) in the focal plane was found long ago $[6,8]$, in terms of the Airy and associated Bi functions:

$$
\Psi_{\mathrm{E}}(x, y, 0)=2 \pi^{2}\left(\frac{2}{3}\right)^{2 / 3} \operatorname{Re}\left[\mathrm{Ai}\left(\frac{x+\mathrm{i} y}{12^{1 / 3}}\right) \mathrm{Bi}\left(\frac{x-\mathrm{i} y}{12^{1 / 3}}\right)\right] .
$$


For later reference, it is convenient to give an alternative expression in terms of the 'one-sided Airy function'

$$
\Psi_{1, \text { inc }}(t) \equiv \int_{0}^{\infty} \mathrm{d} u \exp \left\{\mathrm{i}\left(u^{3}+t u\right)\right\}
$$

(also expressible as the Scorer function $\mathrm{Gi}(t)[11,13]$, and easy to evaluate numerically by rotating the integration contour). Defining

$$
\rho \equiv \frac{x+\mathrm{i} y}{2^{2 / 3}}, \quad e_{ \pm} \equiv \exp \left( \pm \frac{2}{3} \mathrm{i} \pi\right)
$$

the representation is

$$
\begin{aligned}
\Psi_{\mathrm{E}}(x, y, 0)= & 2^{2 / 3} \operatorname{Im}\left[\Psi_{1, \text { inc }}(\rho) \Psi_{1, \text { inc }}\left(\rho^{*}\right)\right. \\
& \left.+\Psi_{1, \text { inc }}\left(e_{-} \rho\right) \Psi_{1, \text { inc }}\left(e_{+} \rho^{*}\right)+\Psi_{1, \text { inc }}\left(e_{+} \rho\right) \Psi_{1, \text { inc }}\left(e_{-} \rho^{*}\right)\right] .
\end{aligned}
$$

For the hyperbolic umbilic (1.5) in the focal plane, the first integral in (1.5) separates trivially, giving

$$
\Psi_{\mathrm{H}}(x, y, 0)=\frac{4 \pi^{2}}{3^{2 / 3}} \operatorname{Ai}\left(\frac{x}{3^{1 / 3}}\right) \operatorname{Ai}\left(\frac{y}{3^{1 / 3}}\right) .
$$

Away from the focal plane, $\Psi_{\mathrm{E}}(x, y, z)$ and $\Psi_{\mathrm{H}}(x, y, z)$ can be represented as convergent power series in $z$, given in section 36.8 of [13].

For the elliptic unfolding of $X_{9}$ in the focal plane (equation (1.6) with $z=0$ ), we need the 'one-sided Pearcey function', analogous to (3.2), namely

$$
\Psi_{2, \text { inc }}(t) \equiv \int_{0}^{\infty} \mathrm{d} u \exp \left\{\mathrm{i}\left(u^{4}+t u\right)\right\},
$$

which is easy to evaluate numerically, even for complex $t$, by rotating the integration contour. With the definitions

$$
\zeta \equiv \frac{x+\mathrm{i} y}{2^{3 / 4}}, \quad f_{ \pm} \equiv \exp \left( \pm \frac{1}{4} \mathrm{i} \pi\right)
$$

the analogue of (3.4) is

$$
\begin{aligned}
X_{\mathrm{E}}(x, y, 0)= & \frac{\mathrm{i}}{\sqrt{2}}\left[-\Psi_{2, \mathrm{inc}}(\zeta) \Psi_{2, \mathrm{inc}}\left(\zeta^{*}\right)-\Psi_{2, \mathrm{inc}}(-\zeta) \Psi_{2, \mathrm{inc}}\left(-\zeta^{*}\right)\right. \\
& -\Psi_{2, \mathrm{inc}}(\mathrm{i} \zeta) \Psi_{2, \mathrm{inc}}\left(-\mathrm{i} \zeta^{*}\right)-\Psi_{2, \mathrm{inc}}(-\mathrm{i} \zeta) \Psi_{2, \mathrm{inc}}\left(\mathrm{i} \zeta^{*}\right) \\
& +\left(\Psi_{2, \mathrm{inc}}\left(f_{-} \zeta\right) \Psi_{2, \mathrm{inc}}\left(f_{+} \zeta^{*}\right)+\Psi_{2, \mathrm{inc}}\left(-f_{-} \zeta\right) \Psi_{2, \mathrm{inc}}\left(-f_{+} \zeta^{*}\right)\right. \\
& \left.\left.+\Psi_{2, \mathrm{inc}}\left(f_{+} \zeta\right) \Psi_{2, \text { inc }}\left(f_{-} \zeta^{*}\right)+\Psi_{2, \text { inc }}\left(-f_{+} \zeta\right) \Psi_{2, \mathrm{inc}}\left(-f_{-} \zeta^{*}\right)\right)^{*}\right]
\end{aligned}
$$

This focal-plane pattern is illustrated in figure 3 .

Away from the focal point $x=y=0, X_{\mathrm{E}}(x, y, 0)$ can be conveniently approximated by the method of stationary phase applied to (1.6):

$$
\begin{aligned}
X_{\mathrm{E}}(r \cos \phi, r \sin \phi, 0) & \approx \frac{2^{1 / 3} \pi}{3 r^{2 / 3}}\left[\exp \left\{-\frac{3 \mathrm{i}}{2^{8 / 3}} r^{4 / 3} \cos \frac{4}{3} \phi\right\}\right. \\
& \left.+\exp \left\{-\frac{3 \mathrm{i}}{2^{8 / 3}} r^{4 / 3} \cos \left(\frac{4}{3} \phi+\frac{2}{3} \pi\right)\right\}+\exp \left\{-\frac{3 \mathrm{i}}{2^{8 / 3}} r^{4 / 3} \cos \left(\frac{4}{3} \phi-\frac{2}{3} \pi\right)\right\}\right]
\end{aligned}
$$

Except for the divergence at the focus $r=0$, this reproduces the intensity pattern in figure 2(a) very well, and generates a phase pattern indistinguishable from figure $2(b)$. A further extension, in the form of a stationary-phase formula for finite $z$, has been published already [7]. 

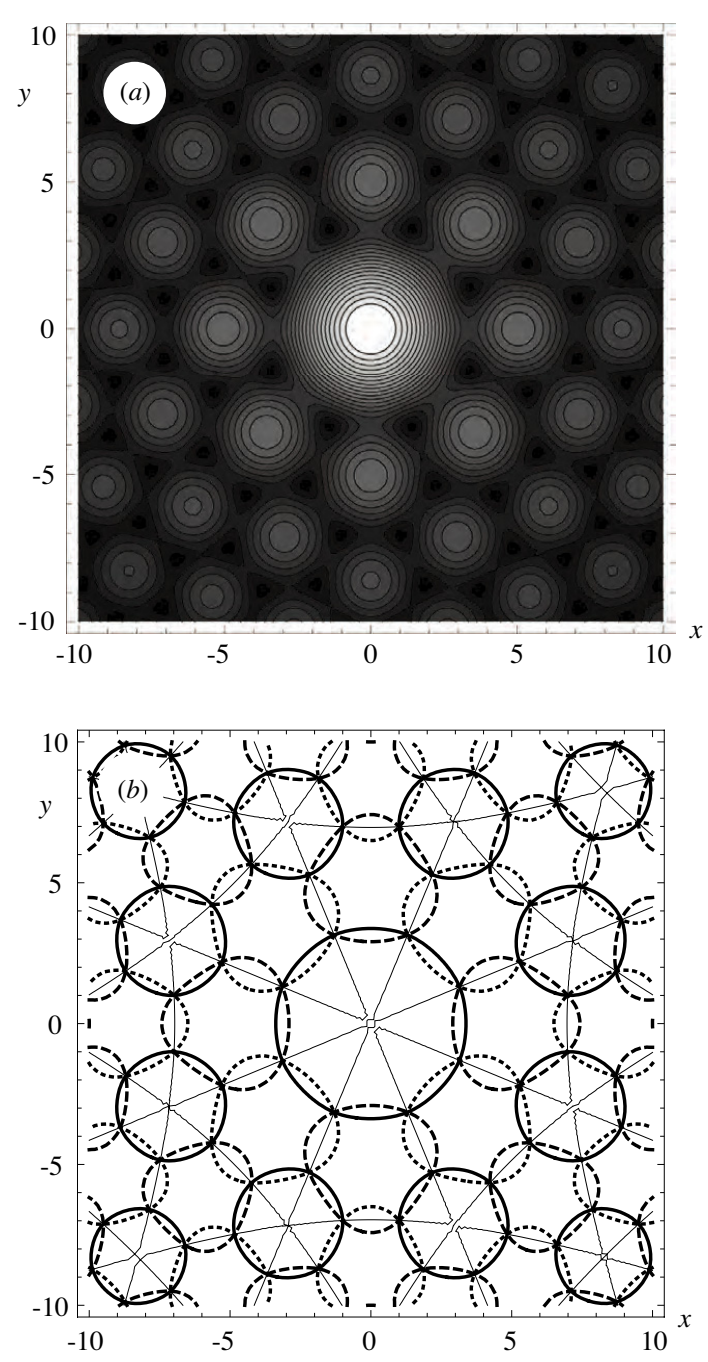

Figure 3. Focal-plane elliptic $X_{9}$ diffraction catastrophe $X_{\mathrm{E}}(x, y, 0)$ (equation (3.8). (a) Modulus; (b) phase contours at intervals of $\pi / 4$.

Away from the focal plane, we can also use the following new convergent power series in $z$, analogous to results in section 36.8 of [13] for $\Psi_{\mathrm{E}}$ and involving the definitions (3.6) and (3.7):

$$
\begin{aligned}
X_{E}(x, y, z)= & E_{-}\left(-i \zeta, i \zeta^{*}\right)+E_{-}\left(\zeta, \zeta^{*}\right)+E_{-}\left(i \zeta,-i \zeta^{*}\right)+E_{-}\left(-\zeta,-\zeta^{*}\right)+ \\
& +\left\{E_{+}\left(f_{-} \zeta, f_{+} \zeta^{*}\right)+E_{+}\left(f_{+} \zeta, f_{-} \zeta^{*}\right)+E_{+}\left(-f_{-} \zeta,-f_{+} \zeta^{*}\right)+E_{+}\left(-f_{+} \zeta,-f_{-} \zeta^{*}\right)\right\}^{*}
\end{aligned}
$$

where

$$
E_{ \pm}(X, Y)=-\frac{\mathrm{i}}{\sqrt{2}} \sum_{r=0}^{\infty} \frac{( \pm \mathrm{i} \sqrt{2} z)^{r}}{r !} E_{r}(X) E_{r}(Y)
$$

and

$E_{r}(t)=a_{r}(t) \Psi_{2, \text { inc }}(t)+b_{r}(t) \partial_{t} \Psi_{2, \text { inc }}(t)+c_{r}(t) \partial_{t}^{2} \Psi_{2, \text { inc }}(t)+d_{r}(t)$, 

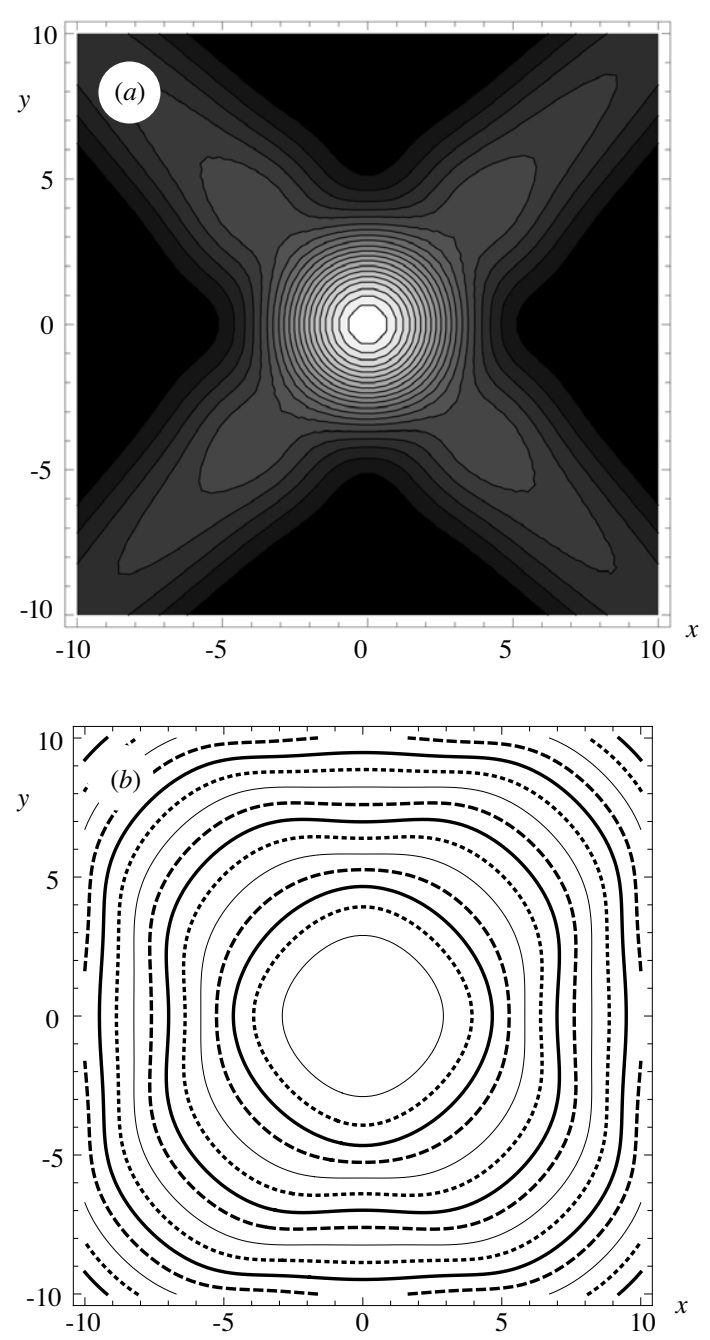

Figure 4. Focal-plane hyperbolic $X_{9}$ diffraction catastrophe $X_{\mathrm{H}}(x, y, 0)$ (equation (3.14). (a) Modulus; $(b)$ phase contours at intervals of $\pi / 4$.

with coefficients generated recursively by

$$
\begin{array}{llrl}
a_{r}(\eta)=\partial_{\eta} a_{r-1}(\eta)+\frac{\mathrm{i} \eta}{4} c_{r-1}(\eta), & b_{r}(\eta)=\partial_{\eta} b_{r-1}(\eta)+a_{r-1}(\eta), \\
c_{r}(\eta)=\partial_{\eta} c_{r-1}(\eta)+b_{r-1}(\eta), & & d_{r}(\eta)=\partial_{\eta} d_{r-1}(\eta)+\frac{1}{4} c_{r-1}(\eta), \\
a_{0}(\eta)=1, & b_{0}(\eta)=0, & c_{0}(\eta)=0, & d_{0}(\eta)=0 .
\end{array}
$$

For the hyperbolic unfolding of $X_{9}$ in the focal plane, the first integral in (1.7) separates when $z=0$, giving the following expression as a product of sections of Pearcey functions, perpendicular to the symmetry axis and passing through the focus:

$$
X_{\mathrm{H}}(x, y, 0)=\frac{1}{\sqrt{2}} \Psi_{2}\left(\frac{x+y}{2^{3 / 4}}, 0\right) \Psi_{2}\left(\frac{x-y}{2^{3 / 4}}, 0\right)
$$

This is illustrated in figure 4. 
Away from the focal plane, we can use a new convergent power series in $z$, analogous to results in section 36.8 of [13] for $\Psi_{\mathrm{H}}$ and involving the definitions

$$
X=\frac{x+y}{2^{3 / 4}}, \quad Y=\frac{x-y}{2^{3 / 4}} .
$$

The series is

$$
X_{H}(x, y, z)=\frac{1}{\sqrt{2}} \sum_{r=0}^{\infty} \frac{(-\mathrm{i} \sqrt{2} z)^{r}}{r !} H_{r}(X) H_{r}(Y)
$$

in which

$$
H_{r}(\eta)=a_{r}(\eta) \Psi_{2}(\eta, 0)+b_{r}(\eta) \partial_{\eta} \Psi_{2}(\eta, 0)+c_{r}(\eta) \partial_{\eta}^{2} \Psi_{2}(\eta, 0)
$$

with coefficients generated recursively by

$$
\begin{aligned}
& a_{r}(\eta)=\partial_{\eta} a_{r-1}(\eta)+\frac{\mathrm{i} \eta}{4} c_{r-1}(\eta), \quad b_{r}(\eta)=\partial_{\eta} b_{r-1}(\eta)+a_{r-1}(\eta), \\
& c_{r}(\eta)=\partial_{\eta} c_{r-1}(\eta)+b_{r-1}(\eta), \quad a_{0}(\eta)=1, \quad b_{0}(\eta)=0, \quad c_{0}(\eta)=0 .
\end{aligned}
$$

\section{Concluding remarks}

It is tempting to speculate that the diffraction catastrophe sections studied here are not the only ones that can be expressed in terms of Bessel functions. For example, we might envisage that the pattern in (1.1) and (1.3) might extend to higher cuspoids, in the form of the sections

$$
\Psi_{n}(z)=\int_{-\infty}^{\infty} \mathrm{d} s \exp \left\{\mathrm{i}\left(s^{n+2}+z s^{n}\right)\right\}
$$

for $n>2$, but this does not seem to be the case. We have explored the case $n=3$ (swallowtail section) in some detail. Some of the oscillatory behaviour can be captured in terms of the Bessel functions $J_{ \pm 1 / 5}$, but it seems that features associated with the degenerate critical point at $s=0$ cannot be so represented.

\section{Acknowledgment}

MVB thanks the Leverhulme Trust for research support, and the University of Pennsylvania for generous hospitality while this paper was written.

\section{Appendix A. Derivation of axial formulas}

First we derive the expression in the last line of (2.1), for the axial elliptic umbilic. The starting point is equation (3.8) of [6] (noting a factor $2 \pi$ difference in the definitions), or, equivalently, formula (36.2.26) of [13] (written as the sum of two integrals):

$$
\Psi_{\mathrm{E}}(0,0, z)=2 \sqrt{\frac{\pi}{3}} \exp \left(\frac{4}{27} \mathrm{i} z^{3}\right)\left(I(z)+I^{*}(-z)\right),
$$

where

$$
I(z)=\exp \left(-\frac{1}{4} \mathrm{i} \pi\right) \int_{0}^{\infty \exp (\mathrm{i} \pi / 12)} \mathrm{d} u \exp \left\{\mathrm{i}\left(u^{6}+2 z u^{4}+z^{2} u^{2}\right)\right\} .
$$

With the change of variable

$$
u=2 \sqrt{\frac{z}{3}} \sinh \frac{t}{6}, \quad \text { i.e. } u^{6}+2 z u^{4}+z^{2} u^{2}=\frac{2}{27} z^{3}(\cosh t-1),
$$


(A.2) becomes

$$
\begin{aligned}
I(z)= & \sqrt{\frac{z}{27}} \exp \left(-\frac{2}{27} \mathrm{i} z^{3}-\frac{1}{4} \mathrm{i} \pi\right) \int_{0}^{\infty+\mathrm{i} \pi / 2} \mathrm{~d} t \exp \left(\frac{2}{27} \mathrm{i} z^{3} \cosh t\right) \cosh \frac{t}{6} \\
= & \frac{\pi}{2} \sqrt{\frac{z}{27}} \exp \left(-\frac{2}{27} \mathrm{i} z^{3}+\frac{1}{3} \mathrm{i} \pi\right) H_{1 / 6}^{(1)}\left(\frac{2}{27} z^{3}\right) \\
= & \pi \sqrt{\frac{z}{27}} \exp \left(-\frac{2}{27} \mathrm{i} z^{3}-\frac{1}{4} \mathrm{i} \pi\right) \\
& \times\left(\exp \left(\frac{1}{12} \mathrm{i} \pi\right) J_{-1 / 6}\left(\frac{2}{27} z^{3}\right)-\exp \left(-\frac{1}{12} \mathrm{i} \pi\right) J_{1 / 6}\left(\frac{2}{27} z^{3}\right)\right),
\end{aligned}
$$

where equation (10.9.10) of [13] has been used to identify the Hankel function, and (10.4.7) to express this in terms of the Bessel functions $J_{ \pm 1 / 6}$. Next, we use $z \rightarrow z \exp (\mathrm{i} \pi)$ and the Bessel continuation formula (10.11.1) of [13], to get

$$
\begin{aligned}
I(-z)=\pi \sqrt{\frac{z}{27}} & \exp \left(\frac{2}{27} \mathrm{i} z^{3}+\frac{1}{4} \mathrm{i} \pi\right) \\
& \times\left(\exp \left(-\frac{5}{12} \mathrm{i} \pi\right) J_{-1 / 6}\left(\frac{2}{27} z^{3}\right)-\exp \left(\frac{5}{12} \mathrm{i} \pi\right) J_{1 / 6}\left(\frac{2}{27} z^{3}\right)\right) .
\end{aligned}
$$

The desired last line of (2.1) now follows from (A.1) after elementary algebra.

Now we derive the expressions in the last two lines of (2.3), for the axial hyperbolic umbilic, starting from formula (36.2.8) of [13]. This involves a variable $u$, and a path from $u=\infty \exp (5 \mathrm{i} \pi / 12)$ via $u=0$ to $u=\infty \exp (\mathrm{i} \pi / 12)$. Writing the integrals separately, and transforming the path from $u=\infty \exp (5 \mathrm{i} \pi / 12)$ to $u=0$ by changing the variable to $u=\mathrm{i} v$, we get

$$
\begin{aligned}
\Psi_{\mathrm{H}}(0,0, z)= & 4 \sqrt{\frac{\pi}{6}} \exp \left(\frac{1}{27} \mathrm{i} z^{3}+\frac{1}{4} \mathrm{i} \pi\right) \\
& \times\left[\int_{0}^{\infty \exp (\mathrm{i} \pi / 12)} \mathrm{d} u \exp \left\{\mathrm{i}\left(2 u^{6}+2 z u^{2}+\frac{1}{2} z^{2} u^{2}\right)\right\}\right. \\
& \left.-\mathrm{i} \int_{0}^{\infty \exp (-\mathrm{i} \pi / 12)} \mathrm{d} v \exp \left\{-\mathrm{i}\left(2 u^{6}-2 z u^{2}+\frac{1}{2} z^{2} u^{2}\right)\right\}\right] .
\end{aligned}
$$

Expressing the integrals in terms of $I(z)$ (equation (A.2)) gives

$$
\Psi_{\mathrm{H}}(0,0, z)=2^{4 / 3} \sqrt{\frac{\pi}{3}} \mathrm{i}\left(I(z)-I^{*}(-z)\right),
$$

whence substitution of (A.4) and (A.5) leads to the expression on the third line of (2.3).

The expression on the last line of (2.3), unexpectedly relating the axial elliptic and hyperbolic integrals, follows from the relation

$$
\sqrt{3} \mathrm{i} \exp \left(\frac{4}{27} \mathrm{i} z^{3}\right) \frac{\left(I(z)-I^{*}(-z)\right)}{\left(I^{*}(z)-I(-z)\right)}=1
$$

which itself can be derived from (A.4) and (A.5). We have not succeeded in deriving (A.8) directly from the integral representation (A.2).

Next, we derive the expression on the last line of (2.4), for the axial elliptic $X_{9}$ integral. Substituting $\rho=\sqrt{x}$ in the second line gives

$$
X_{\mathrm{E}}(0,0, z)=\frac{1}{2} \pi \int_{0}^{\infty} \frac{\mathrm{d} x}{\sqrt{x}} \exp (\mathrm{i} z \sqrt{x}) J_{0}(x) .
$$


We evaluate this by combining formulas (6.753.3) and (6.753.4) of [15], to get

$$
\int_{0}^{\infty} \frac{\mathrm{d} x}{\sqrt{x}} \exp \{a \sqrt{x}(\mathrm{i}-1)\} J_{0}(x)=\frac{1}{2} a K_{1 / 4}\left(\frac{1}{4} a^{2}\right)\left[I_{-1 / 4}\left(\frac{1}{4} a^{2}\right)+\mathrm{i} I_{1 / 4}\left(\frac{1}{4} a^{2}\right)\right] \text {. }
$$

Then the substitution $a=z \exp \left(-\frac{1}{4} \mathrm{i} \pi\right) / \sqrt{2}$ leads to the expression in the last line of (2.4) after using standard continuation formulas to express the modified Bessel functions $K$ and $I$ in terms of the $J$ Bessel functions.

Next, we derive the expressions (2.6), for the axial hyperbolic $X_{9}$ integral. Substituting $\rho=\sqrt{x}$ in the third line, and using a special case of formula (6.734) of [15], we get

$$
X_{\mathrm{H}}(0,0, z)=\frac{\pi}{2} \exp \left(\frac{1}{4} \mathrm{i} \pi\right) D_{-1 / 2}\left(\frac{z}{\sqrt{2}} \exp \left(\frac{1}{4} \mathrm{i} \pi\right)\right) D_{-1 / 2}\left(-\frac{z}{\sqrt{2}} \exp \left(\frac{1}{4} \mathrm{i} \pi\right)\right),
$$

where $D$ denotes the parabolic cylinder function. These can be expressed in terms of modified Bessel functions by successively using formulas (12.1), (12.7.10) and (12.2.15) of [13], and (19.15.13) of [11], leading to

$$
X_{\mathrm{H}}(0,0, z)=\frac{1}{8} \pi \mathrm{i} z K_{1 / 4}\left(\frac{1}{8} \mathrm{i} z^{2}\right)\left[I_{1 / 4}\left(\frac{1}{8} \mathrm{i} z^{2}\right)+I_{-1 / 4}\left(\frac{1}{8} \mathrm{i} z^{2}\right)\right] .
$$

The expression in the fourth line of (2.6) now follows from standard continuation formulas to express the modified Bessel functions $K$ and $I$ in terms of the $J$ Bessel functions.

The expression on the last line of (2.6), unexpectedly relating the $X_{\mathrm{E}}$ and $X_{\mathrm{H}}$ integrals, follows from the $J$ Bessel expressions in the third line of (2.4) and the fourth line of (2.6), and standard relations between the Bessel and Hankel functions. We have not succeeded in deriving the relation between $X_{\mathrm{E}}$ and $X_{\mathrm{H}}$ directly from the integral representations in the second line of (2.4) and the third line of (2.6).

\section{Appendix B. Derivation of focal-plane formulas}

First we derive equation (3.4) for the elliptic umbilic integral (1.4) in the focal plane. In polar coordinates,

$$
\Psi_{\mathrm{E}}(x, y, 0)=\int_{0}^{\infty} \mathrm{d} r r \int_{0}^{2 \pi} \mathrm{d} \theta \exp \left\{\mathrm{i}\left(r^{3} \cos 3 \theta+r(x \cos \theta+y \sin \theta)\right)\right\} .
$$

The transformation $\theta \rightarrow \theta+\pi$ shows that this is real, so we can divide the angular range into any set of subintervals whose complement is generated by a $\pi$ rotation. It is convenient to choose

$$
-\frac{1}{6} \pi<\theta<\frac{1}{6} \pi, \quad \frac{1}{2} \pi<\theta<\frac{5}{6} \pi, \quad-\frac{5}{6} \pi<\theta<-\frac{1}{2} \pi,
$$

so that

$$
\begin{aligned}
\Psi_{\mathrm{E}}(x, y, 0)= & 2 \operatorname{Re} \int_{0}^{\infty} \mathrm{d} r r \int_{-\pi / 6}^{\pi / 6} \mathrm{~d} \theta \exp \left(\mathrm{i} r^{3} \cos 3 \theta\right)[\exp (\mathrm{i} r(x \cos \theta+y \sin \theta)) \\
& +\exp \left(\mathrm{i} r\left(x \cos \left(\theta-\frac{2}{3} \pi\right)+y \sin \left(\theta-\frac{2}{3} \pi\right)\right)\right) \\
& \left.+\exp \left(\mathrm{i} r\left(x \cos \left(\theta+\frac{2}{3} \pi\right)+y \sin \left(\theta+\frac{2}{3} \pi\right)\right)\right)\right] .
\end{aligned}
$$

With this choice, the integrals converge for all $\theta$ if the $r$ contour is deformed to $|r| \exp \left(\frac{1}{6} \mathrm{i} \pi\right)$. Now we define new integration variables $u$ and $v$ by

$$
\begin{aligned}
& s=r \cos \theta=\frac{1}{2^{2 / 3}}(u+v), \quad t=r \sin \theta=\frac{\mathrm{i}}{2^{2 / 3}}(u-v), \\
& \text { i.e. } u=\frac{r}{2^{1 / 2}} \exp (-\mathrm{i} \theta), \quad v=\frac{r}{2^{1 / 2}} \exp (\mathrm{i} \theta)
\end{aligned}
$$


chosen because

$$
r^{3} \cos 3 \theta+r(x \cos \theta+y \sin \theta)=u^{3}+v^{3}+\rho u+\rho^{*} v
$$

where $\rho$ is defined in (3.3). With the deformed $r$ contour, the $u$ and $v$ contours for given $\theta$ extend from the origin into sectors with $\arg (u) \leqslant \pi / 3$ and $\arg (v) \leqslant \pi / 3$, which can be deformed to the real axis while preserving convergence. Then the $u$ and $v$ integrals separate, and can be identified as the one-sided Airy function (3.2), and (3.4) follows immediately from (B.3).

Now we derive equation (3.8) for the elliptic $X_{9}$ integral (1.6) in the focal plane. In polar coordinates,

$X_{\mathrm{E}}(x, y, 0)=\int_{0}^{\infty} \mathrm{d} r r \int_{0}^{2 \pi} \mathrm{d} \theta \exp \left\{\mathrm{i}\left(r^{4} \cos 4 \theta+r(x \cos \theta+y \sin \theta)\right)\right\}$.

The argument is similar to that just given for $\Psi_{\mathrm{E}}(x, y, 0)$, except that now the function is not real. Instead, we divide the range of integration into eight intervals, centred on $\theta=n \pi / 4(-3$ $\leqslant n \leqslant 4) \cdot \cos 4 \theta$ has the same sign in alternating intervals, leading to

$$
\begin{aligned}
X_{\mathrm{E}}(x, y, 0)= & \int_{0}^{\infty} \mathrm{d} r r \int_{-\pi / 8}^{\pi / 8} \mathrm{~d} \theta \exp \left(\mathrm{i} r^{4} \cos 4 \theta\right) \times \\
& \sum_{n=-1}^{2} \exp \left\{\mathrm{i} r\left(x \cos \left(\theta-\frac{1}{2} n \pi\right)+y \sin \left(\theta-\frac{1}{2} n \pi\right)\right)\right\}+ \\
& {\left[\int_{0}^{\infty} \mathrm{d} r r \int_{-\pi / 8}^{\pi / 8} \mathrm{~d} \theta \exp \left(\mathrm{i} r^{4} \cos 4 \theta\right) \sum_{n=-1}^{2} \times\right.} \\
& \left.\exp \left\{\mathrm{i} r\left(x \cos \left(\theta-\frac{1}{2}\left(n+\frac{1}{2}\right) \pi\right)+y \sin \left(\theta-\frac{1}{2}\left(n+\frac{1}{2}\right) \pi\right)\right)\right\}\right]^{*} .
\end{aligned}
$$

The integrals converge for all $\theta$ if the $r$ contour is deformed to $|r| \exp \left(\frac{1}{8} \mathrm{i} \pi\right)$. Now we define (cf. B.4)

$$
\begin{aligned}
& s=r \cos \theta=\frac{1}{2^{3 / 4}}(u+v), \quad t=r \sin \theta=\frac{\mathrm{i}}{2^{3 / 4}}(u-v), \\
& \text { i.e. } u=\frac{r}{2^{1 / 4}} \exp (-\mathrm{i} \theta), \quad v=\frac{r}{2^{1 / 4}} \exp (\mathrm{i} \theta),
\end{aligned}
$$

chosen because

$$
r^{4} \cos 4 \theta+r(x \cos \theta+y \sin \theta)=u^{4}+v^{4}+\zeta u+\zeta^{*} v
$$

where $\zeta$ is defined in (3.7). With the deformed $r$ contour, the $u$ and $v$ contours for given $\theta$ extend from the origin into sectors with $\arg (u) \leqslant \pi / 4$ and $\arg (v) \leqslant \pi / 4$, which can be deformed to the real axis while preserving convergence. Then the $u$ and $v$ integrals separate, and can be identified as the one-sided Pearcey function (3.6), and (3.8) follows immediately from (B.7).

The derivations of the convergent series (3.10-3.13) for $X_{\mathrm{E}}$ and (3.15-3.18) for $X_{\mathrm{H}}$ are straightforward, though it is worth noting the following inhomogeneous differential equation, used in the derivation of the series for $X_{\mathrm{E}}$ :

$$
\partial_{t}^{3} \Psi_{2, \text { inc }}(t)-\frac{\mathrm{i}}{4} t \Psi_{2, \text { inc }}(t)=\frac{1}{4} \text {. }
$$




\section{References}

[1] Poston T and Stewart I 1978 Catastrophe Theory and its Applications (London: Pitman (reprinted by Dover))

[2] Berry M V and Upstill C 1980 Catastrophe optics: morphologies of caustics and their diffraction patterns Prog. Opt. 18 257-346

[3] Nye J F 1999 Natural Focusing and Fine Structure of Light: Caustics and Wave Dislocations (Bristol: Institute of Physics Publishing)

[4] Nye J F 1986 The catastrophe optics of liquid drop lenses Proc. R. Soc. Lond. A 403 1-26

[5] Nye J F 2006 Dislocation lines in the hyperbolic umbilic diffraction catastrophe Proc. R. Soc. A 462 2299-313

[6] Berry M V, Nye J F and Wright F J 1979 The elliptic umbilic diffraction catastrophe Phil. Trans. R. Soc. A 291 453-84

[7] Nye J F 2010 Wave dislocations in the diffraction pattern of a higher-order catastrophe J. Opt. 12015702

[8] Trinkaus H and Drepper F 1977 On the analysis of diffraction catastrophes J. Phys. A 10 L-11-L16

[9] Airy G B 1838 On the intensity of light in the neighbourhood of a caustic Trans. Camb. Phil. Soc. 6 379-403

[10] Vallée O and Soares M 2004 Airy Functions and Applications to Physics (London: Imperial College Press)

[11] Abramowitz M and Stegun I A 1972 Handbook of Mathematical Functions (Washington: National Bureau of Standards)

[12] Berry M V 2001 Why are special functions special? Phys. Today April 11-12

[13] DLMF 2010 NIST Handbook of Mathematical Functions (Cambridge: University Press) http://dlmf.nist.gov

[14] Pearcey T 1946 The structure of an electromagnetic field in the neighbourhood of a cusp of a caustic Phil. Mag. 37 311-7

[15] Gradshteyn I S and Ryzhik I M 1980 Table of Integrals, Series and Products (New York: Academic) 